\title{
Efeito imediato da MWM de Mulligan em pacientes com dor no ombro
}

\author{
MWM Mulligan immediate effect on shoulder pain patients
}

\author{
Daniela Dias da Silva Garzedin ${ }^{1 *}$, Vítor Beltrão ${ }^{2}$, Cesar Diniz ${ }^{3}$, Matheus Henrique Almeida da Silva ${ }^{4}$, \\ Fernanda dos Santos Régis ${ }^{4}$, Roberto Paulo Correia de Araújo ${ }^{5}$
}

\begin{abstract}
${ }^{1}$ Doutoranda da Pós-Graduação em Processos Interativos de Órgãos e Sistemas, Instituto de Ciências da Saúde, UFBA. Professora Adjunto I do Departamento de Fisioterapia da UFBA.; ${ }^{2}$ Fisioterapeuta com especialização na área de esporte; ${ }^{3}$ Fisioterapeuta da Clínica Escola de Fisioterapia da UFBA.; ${ }^{4}$ Graduando do Curso de Fisioterapia da UFBA.; ${ }^{5}$ Professor Titular Livre Docente de Bioquímica. Instituto de Ciências da Saúde, UFBA.
\end{abstract}

\begin{abstract}
Resumo
Introdução: a dor no ombro leva a limitações e incapacidades entre adultos e idosos, merecendo atenção do fisioterapeuta. Objetivo: Avaliar o efeito imediato da técnica de Mulligan em pacientes com dor no ombro. Metodologia: trata-se de resultados preliminares de um ensaio clínico realizado entre julho de 2018 a julho de 2019. Pacientes com dor no ombro $(n=44)$, de ambos os sexos, foram randomizados e submetidos a dois protocolos de tratamento: exercícios terapêuticos ( $n=22)$ e MWM de Mulligan ( $n=22)$, atendidos na Clínica Escola de Fisioterapia da UFBA. Todos os participantes responderam a um questionário clínico e sociodemográfico e realizaram avaliação da dor pela escala visual analógica (EVA) e algometria. Os pacientes foram tratados por dois fisioterapeutas experientes na área e, depois, foram reavaliados imediatamente. Resultados: mulheres predominaram, a média de idade foi de 56 anos no grupo Mulligan e 57 no grupo exercícios terapêuticos. Síndrome do manguito rotador predominou: 19 (86,4\%) no grupo Mulligan e 15 (68\%) no grupo exercícios. O ombro mais acometido foi o direito no Grupo Mulligan, (17 (77,3\%); já no grupo exercícios, foi o esquerdo com $12(55 \%)$. A cor negra foi a mais autorrelatada pelos pacientes, e poucos tinham o ensino superior completo. No grupo Mulligan, as ocupações mais informadas foram dona de casa, seguida de aposentados e autônomos. No grupo exercícios, não houve dona de casa, e sim mais aposentados e autônomos. Os dois grupos mostraram ser mais sedentários. Não houve diferença da dor pela EVA entre os grupos, antes e após o tratamento ( $p=0.79, p=0.56$, respectivamente). Já a intensidade da dor mensurada pela algometria, tanto antes do tratamento $(p=0.008)$ quanto depois do tratamento $(p=0.04)$, foi diferente entre os grupos, com predomínio de melhora no grupo Exercícios. Conclusão: a MWM não foi mais eficaz que os exercícios terapêuticos na redução da dor do ombro, sendo que o exercício apresentou mais eficácia quando medido através da EVA e sem melhora significativa ao ser mensurada pela algometria após aplicação de uma única sessão de tratamento.
\end{abstract}

Palavras-chave: Técnica de Mulligan. Cinesiologia Aplicada. Dor no ombro.

\begin{abstract}
Introduction: shoulder pain leads to limitations and disabilities among adults and the elderly deserving attention from the physiotherapist. Objective: to evaluate the immediate effect of the Mulligan technique in patients with shoulder pain. Methodology: these are preliminary results of a clinical trial conducted from July 2018 to July 2019. Patients $(n=44)$ with shoulder pain of both sexes were randomized and submitted to two treatment protocols: therapeutic exercises $(n=22)$ and Mulligan's MWM $(n=22)$ attended at the UFBA School of Physiotherapy Clinic. All participants answered a clinical and sociodemographic questionnaire and underwent pain assessment by visual analog scale (VAS) and algometry. The patients were treated by two experienced physiotherapists and then reassessed immediately. Results: women predominated, the average age was 56 years in the Mulligan group and 57 in the therapeutic exercises group. Rotator cuff syndrome predominated, $19(86.4 \%)$ in the Mulligan group and $15(68 \%)$ in the exercise group. The most affected shoulder was the right shoulder in the Mulligan Group (17 (77.3\%), while in the exercise group was the left shoulder with 12 (55\%).The black color was the most self-reported by the patients and few patients had higher education. In the Mulligan group the most informed occupations were housewife followed by retirees and self-employed. In the exercise group we had no housewife but more retired and self-employed. The two groups were more sedentary. There was no difference in VAS pain between the groups before and after treatment ( $p=0.79, p=0.56$, respectively), while pain intensity measured by algometry both before treatment ( $p$ $=0.008$ ) and after treatment ( $p=0.04)$ was different between Conclusion: Mulligan's MWM was not more effective than exercise in reducing shoulder pain, and exercise was more effective when measured by VAS and without significant improvement when measured by algometry after applying a single treatment session.
\end{abstract}

Kewyords: Kinesiology Applied. Mulligan Technique. Shoulder pain.

\section{INTRODUÇÃO}

Dor no ombro é uma experiência comum entre

Correspondente/Corresponding: *Daniela Dias da Silva Garzedin Avenida Reitor Miguel Calmon S/N. Canela Campus. Salvador, BA. CEP: 40.110-903 - Tel: + 55 (71) 3283-8901 - E-mail: ddsgarzedin@yahoo. com.br. trabalhadores adultos e também entre idosos e, como disfunção incapacitante, merece atenção do fisioterapeuta. Apresenta prevalência relatada entre 20 a 33\% na população adulta, podendo esse número aumentar em idosos por questões de envelhecimento biológico (LUIME et al., 2004).

A dor no ombro pode ser decorrente de desarranjos 
biomecânicos, sendo considerada disfunção musculoesquelética multifatorial, secundária a lesões em estruturas articulares, ósseas, cartilaginosas e musculotendíneas (XU et al., 2015). Dias et al. estudaram ombro doloroso em 77 indivíduos e verificaram que a síndrome do manguito rotador foi a etiologia que predominou, sendo que a dor era frequente e mais intensa em mulheres e na faixa etária entre 40 e 65 anos (DIAS et al., 2008). Em outro estudo realizado pelos mesmos autores com 103 pacientes acometidos pela Síndrome do MR, encontraram predominância de dor em ombro direito, dominante, e o perfil da dor mostrou-se de intensidade leve a moderada, dor ao movimento, mais frequente durante a noite e de longa duração (DIAS et al., 2016).

As dores no ombro configuram um problema de saúde tanto do trabalhador como da população geral, que lida com movimentos repetitivos, muitas vezes acima da cabeça, com repercussões econômicas e sociais, principalmente quando associadas às incapacidades funcionais, atingindo a atividade produtiva e promovendo afastamento do trabalho. (MENDONÇA JÚNIOR; ASSUNÇÃO, 2005).

Programas de exercícios terapêuticos direcionados ao ombro têm evidenciado bons resultados para o alívio da dor, para restabelecer as amplitudes articulares de movimento e também para o equilíbrio, o aumento da força muscular e da função. Porém técnicas de terapia manual, combinadas com exercícios terapêuticos, parecem produzir melhor controle da dor e ganho de funcionalidade superior à do exercício terapêutico isolado (KISNER; CAROLYN; COLBY, 2015; MULLIGAN, 2006; YO; SOLE; MUNN, 2009).

A mobilization with movement (MWM), de Brian Mulligan, é uma técnica de terapia manual que se baseia na teoria da falha posicional articular e também tem indicação de reduzir dor e ganhar arco de movimento. Segundo Mulligan, diante das lesões do ombro, vai ocorrer anteriorização e superiorização da cabeça umeral, reduzindo o espaço subacromial e causando bloqueio do movimento. Através do glide, deslizamento da cabeça umeral, objetiva-se o reposicionamento articular, contribuindo para um movimento biomecanicamente correto, proporcionando alívio da dor e ganho da amplitude de movimento do ombro de forma imediata. (MULLIGAN, 2009; VICENZINO; PAUNGMALI; TEYS, 2007; WRIGHT, 1995).

De acordo com Mulligan, em uma única sessão de tratamento, é possível conseguir redução imediata da dor e ganho da ADM do ombro. Patrício (2013) avaliou 24 indivíduos randomizados em dois grupos. Um grupo utilizou a técnica MWM, e o outro o placebo, sendo que, nesse grupo, os movimentos foram realizados ativamente com o fisioterapeuta posicionando as mãos no ombro no mesmo local da técnica MWM, porém sem realizar glide. Os autores concluíram que a MWM reduziu a dor nos indivíduos com síndrome do impacto, aumentou a ADM do ombro até o início da dor e sugerem a utilização dessa técnica antes de iniciar o exercício terapêutico, devido à inibição da dor e para prolongar padrões de correção automática induzidas pelo MWM.
Já Guimarães, Salvini e Siqueira (2016) não encontraram eficácia imediata da MWM se comparado a sham para o aumento da ADM de rotação externa e da abdução, melhora da dor e função do ombro em 27 indivíduos com síndrome do impacto do ombro. Além disso, ambas as técnicas se mostraram ineficazes para ganho de flexão anterior e força muscular.

Teys, Bisset e Vicenzino (2008) e Teys et al. (2013) avaliaram os efeitos da MWM na melhora da dor e no ganho de ADM do ombro. O primeiro estudou 24 participantes com dor no ombro, por três sessões realizadas aproximadamente na mesma hora, e constatou que a técnica MWM, em comparação com a intervenção sham, foi melhor. Já o segundo estudo, feito com 25 indivíduos, avaliou os efeitos da MWM ao longo do tempo, com uma única sessão de tratamento em indivíduos com dor no ombro, e os comparou com os da MWM associada ao tape. Como resultado, observou-se que uma única sessão de MWM produziu melhora na dor e, quando associada ao tape, melhorou também a ADM do ombro, que perdurou até uma semana (TEYS et al., 2013).

Nota-se que são poucos os estudos realizados, e que não existe uma opinião unânime com relação ao efeito imediato da MWM e ao tempo de duração desses efeitos em indivíduos com dor no ombro. Portanto, este estudo tem por objetivo avaliar o efeito imediato da MWM em pacientes com dor no ombro.

\section{METODOLOGIA}

Esse estudo apresenta resultados preliminares do ensaio clínico intitulado "Estudo comparativo de duas técnicas de intervenção fisioterapêutica para dor limitação da função do ombro e qualidade de vida em humanos: um ensaio clínico randomizado". A pesquisa foi realizada entre 20 de julho de 2019 a 20 de dezembro de 2019 na Clínica Escola de Fisioterapia da Universidade Federal da Bahia (UFBA) localizada na Rua Padre Feijó, 312, Canela, Salvador, Bahia, Brasil. O estudo foi cadastrado no REBEC (Registro Brasileiro de Ensaios Clínicos) com n ${ }^{\circ}$ UTN: U 1111-1220-9766, e seguiu as recomendações CONSORT 2010 (Consolidated Standards of Reporting). (MOHER, 2001). O estudo foi aprovado pelo Conselho de Ética em Pesquisa do Instituto de Ciências da Saúde (ICS) (CAAE 55556816.7 .0000 .5028 ) e realizado de acordo com a Declaração de Helsinque. Um Termo de Consentimento Livre e Esclarecido foi obtido de todos os pacientes incluídos na pesquisa.

Participaram do estudo pacientes adultos com dor no ombro encaminhados da Clínica Escola de Fisioterapia da Universidade Federal da Bahia e do Hospital Universitário Magalhães Neto.

Para serem incluídos neste estudo, os participantes tinham de ter idade acima de 18 anos, estar ou não em uso de medicamentos para dor, com dor no ombro, com ou sem hipomobilidade. Ambos os sexos foram contemplados.

Rev. Ciênc. Méd. Biol., Salvador, v. 19, n. 2, p. 335-341, mai./ago. 2020 
Foram adotados como critérios de exclusão: o paciente não suportar as técnicas de tratamento em estudo, com piora da dor por três sessões consecutivas, ou ter mais que $30 \%$ de faltas. E foram tomados como critérios de não inclusão: ter usado corticoide ou feito fisioterapia nos últimos seis meses, ter realizado cirurgia no ombro, ter fraturado o ombro, ser portador de artrite reumatoide, de sequela de AVE, cardiopatia, déficit cognitivo e incapacidade de locomoção e transferências.

O cálculo do tamanho de amostra foi baseado na diferença de médias padronizadas ( $d$ de Cohen) assumindo-se um valor médio final esperado de 2 com desvio padrão (DP) de 0.5 no grupo Mulligan (grupo experimento, GE), uma média final esperada de 3 com DP de 1.5 no grupo cinesioterapia (grupo de controle, GC), para um nível de significância de $0.05 \%$ e poder de teste de $80 \%$, obtendo-se o valor total de amostra de 44 pacientes, 22 em cada grupo.

Os participantes passaram por um processo de randomização para alocação em grupo MWM de Mulligan ou em grupo a ser submetido a exercícios terapêuticos. Portanto, seguiu-se a ordem de alocação da lista randômica, calculada estatisticamente às cegas para avaliações e tratamentos.

Os pacientes foram avaliados por meio de um questionário com informações acerca de dados clínicos e sociodemográficos, como sexo, idade, diagnóstico médico, ombro acometido, dominância, exames de imagem, medicação para dor, medicação para depressão, prática de atividade física, cor da pele, estado civil, escolaridade, situação ocupacional, características da dor.

Nosso estudo utilizou a EVA para a medida da dor por ser uma escala unidimensional simples, sensível, reprodutível e largamente utilizada na prática do fisioterapeuta, permitindo a análise contínua da dor, e ainda por ser um método já estabelecido e validado na literatura. Neste estudo, utilizou-se também a algometria para a avaliação da dor no ombro, por ser um exame com maior acurácia e também por ser considerado padrão ouro na avaliação da dor musculoesquelética (PAUL et al., 2012; TEYS et al., 2013).

A EVA é uma escala validada internacionalmente e mundialmente utilizada. Ela vai de zero (sem dor) a 10 (pior dor imaginável). O paciente foi questionado a informar o valor da sua dor no momento da avaliação e imediatamente após o tratamento.

Para avaliação da dor também foi aplicada a algometria por pressão que é realizada sobre o ponto mais doloroso na região do ombro, indicado pelo paciente e constatada à palpação pelo avaliador. Com o aparelho posicionado perpendicularmente à região dolorosa, uma pressão foi exercida através da sonda de $1 \mathrm{~cm}^{2}$ sobre a pele. A pressão utilizada foi de $40 \mathrm{~K} \mathrm{~Pa} / \mathrm{s}$, e a intensidade foi aumentada gradativamente de 5 em $5 \mathrm{kgf}$. Três medidas repetidas foram registradas, e a média foi calculada para fins de análises de dados (PAUL et al., 2012). De acordo com Walton et al. (2011), apud Teys et al. (2013), esse instrumento tem demonstrado uma boa confiabilidade inter e intraobservador, além de correlação com outras medidas de dor (TEYS et al., 2013).

Ao final das avaliações, todos os pacientes receberam uma cartilha educativa composta por 10 itens com orientações escritas e ilustradas, confeccionada pelo próprio autor, baseada na prática clínica e da literatura, com informações preventivas e posturais para evitar a dor no ombro.

Após passar pela avaliação, os pacientes foram encaminhados para o tratamento através da MWM ou dos exercícios terapêuticos e, em seguida, foram reavaliados imediatamente após o tratamento. Os participantes eram encaminhados às cegas para os fisioterapeutas, a fim de realizar um dos tratamentos.

Antes de dar início ao estudo, o pesquisador responsável realizou a calibração intra e interavaliadores e fisioterapeutas. Os protocolos de tratamento foram elaborados por dois fisioterapeutas experientes na área.

O GE realizou a técnica de MWM para os movimentos de flexão, abdução, rotação interna e rotação externa do ombro. O paciente permanecia em ortostase nos movimentos de flexão, abdução e rotação interna e em decúbito dorsal durante a rotação externa. Com a região tenar da mão do fisioterapeuta posicionada na porção anterior da cabeça umeral do paciente, foi realizado um glide póstero-lateral (deslizamento) da cabeça do úmero, respeitando-se o eixo articular do ombro, durante a realização dos movimentos ativos de flexão, abdução e rotação externa.

Na realização da técnica da MWM para a rotação interna, o paciente se encontrava em ortostase, com a mão do correspondente ombro doloroso atrás das costas, segurando a faixa inelástica do Mulligan, e com a mão contralateral segurando a faixa acima da cabeça, para poder auxiliar no movimento de rotação interna do ombro. O fisioterapeuta realizava um glide inferior da cabeça umeral com uma mão localizada na região supracondileana, enquanto a outra mão dava um suporte na região infra-axilar, para impedir que houvesse compensação por parte da escápula.

Foram realizadas três séries de 10 repetições para cada movimento, mantidas durante seis segundos, com duração de 30 minutos. Durante a aplicação do glide da cabeça umeral e concomitantemente à realização do movimento ativo pelo paciente, a dor foi aliviada e prosseguiu-se com a técnica. Caso a dor piorasse, o tratamento deveria ser interrompido imediatamente.

O GC realizou exercícios terapêuticos com os mesmos movimentos que foram indicados para o GE. Os exercícios ativos livres foram compostos por três séries de 10 repetições de cada movimento com duração de 30 minutos. O paciente recebeu comando verbal pelo fisioterapeuta e auxílio visual através de um espelho para realizar os movimentos ativos do ombro. Correções realizadas pelo fisioterapeuta foram feitas quando necessárias. Foram definidas como limites para interrupção dos movimentos ativos livres a piora da dor, a limitação por estiramento 
das partes moles ou a rigidez articular do ombro e compensações por parte do paciente secundárias à dor.

Para a apresentação dos resultados, utilizaram-se estatísticas de medidas de tendência central e dispersão para variáveis contínuas, como média e desvio padrão. As variáveis categóricas foram apresentadas sob a forma de percentual. Para comparação de duas médias, foi utilizado o teste $t$ de Student. Foi utilizado o software SPSS for Windows ${ }^{\circledR}$ (versão 15.0) para tabulação e análise dos dados.

\section{RESULTADOS}

Foram avaliados 44 pacientes com dor no ombro e tratados pelas técnicas de MWM de Mulligan e exercícios terapêuticos na Cínica Escola de Fisioterapia da UFBA no período de julho de 2018 a junho de 2019. Não cumpriram os critérios de inclusão 47 pacientes, e houve uma perda de 19 pacientes, dos quais 14 desistiram por motivos pessoais ou por causa de trabalho; cinco foram excluídos, três por excesso de faltas ao tratamento e dois por não suportarem as técnicas e cursar com piora da dor. O fluxo dos pacientes deste estudo está representado na Figura 1.

Figura 1 - Fluxo e progressão dos participantes no estudo

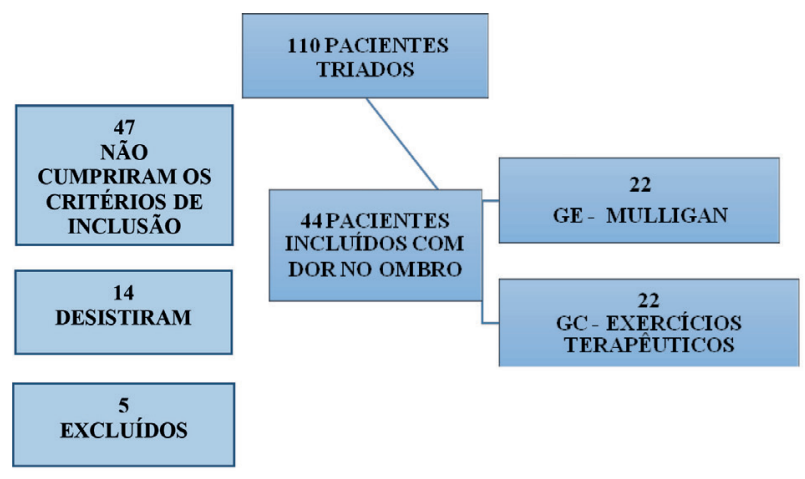

Fonte: Autoria própria

Dos 44 pacientes incluídos no estudo, 22 foram tratados pela MWM de Mulligan e 22 por exercícios terapêuticos, utilizando-se dos mesmos movimentos de flexão, abdução, rotação interna e externa do ombro. Quanto aos dados clínicos e sociodemográficos, observamos que as mulheres predominaram em ambos os grupos, e a média de idade foi semelhante entre os grupos: no grupo Mulligan, 56 anos, e, no grupo de exercícios, 57 anos. De forma geral, o diagnóstico médico mais frequente foi a síndrome do manguito rotador, CID-10 M 75.1, e o ombro esquerdo prevaleceu como o mais acometido no grupo exercícios terapêuticos, sendo a dominância mais frequente à direita. A maioria dos pacientes realizou algum tipo de exame radiológico, como radiografia, ultrassonografia ou ressonância magnética (Tabela 1 ).
Tabela 1 - Caracterização clínico-demográfica de 44 pacientes com dor no ombro em Salvador, Bahia

\begin{tabular}{|c|c|c|}
\hline & GE & GC \\
\hline \multicolumn{3}{|l|}{ Gênero } \\
\hline Feminino & $17(77 \%)$ & $13(60 \%)$ \\
\hline Masculino & $5(23 \%)$ & $9(40 \%)$ \\
\hline Idade (média) & 56 anos (DP 11,7) & 57 anos (DP 11,4) \\
\hline Diagnóstico Médico (CID-10) & $19(86,36)$ & $15(68 \%)$ \\
\hline $\begin{array}{l}\text { M } 75.1 \\
\quad \text { Outros }\end{array}$ & $2(13,63)$ & $7(32 \%)$ \\
\hline \multicolumn{3}{|l|}{ Ombro acometido } \\
\hline Direito & $17(77,3)$ & $10(45 \%)$ \\
\hline Esquerdo & $5(22,7)$ & $12(55 \%)$ \\
\hline \multicolumn{3}{|l|}{ Dominância } \\
\hline Direita & $19(86,4)$ & $21(95 \%)$ \\
\hline Esquerda & $3(13,6)$ & $1(5 \%)$ \\
\hline \multicolumn{3}{|l|}{$\begin{array}{l}\text { Exames Radiológicos (RX, US } \\
\text { ou RM) }\end{array}$} \\
\hline Realizou & $20(90 \%)$ & $19(86 \%)$ \\
\hline Não realizou & $2(10 \%)$ & $3(14 \%)$ \\
\hline
\end{tabular}

GE: grupo experimental; GC: grupo controle; CID-10: código internacional de doenças; RX: raios-x; US: ultrassom; RM: ressonância magnética.

Fonte: Dados da pesquisa.

A Tabela 2 apresenta o perfil social dos pacientes deste estudo. Pudemos observar que a cor de pele mais frequentemente autorrelatada pelo paciente foi cor negra, seguida de mulata e outras.

Com relação à escolaridade, dos 44 pacientes estudados, 6 tinham o ensino superior completo, sendo 4 (18,2\%) do grupo Mulligan e 2 do grupo exercícios.

No grupo Mulligan, as ocupações mais frequentes foram a de dona de casa, seguida de aposentado e autônomo. Entretanto, no grupo de exercícios, não havia donas de casa, e sim mais aposentados e autônomos. Os dois grupos mostraram-se mais sedentários.

Tabela 2 - Perfil social de 44 pacientes com dor no ombro em Salvador, Bahia

\begin{tabular}{lrr}
\hline & n GE (\%) & n GC (\%) \\
\hline Cor da pele & & \\
Branca & $2(9)$ & $1(4,5)$ \\
Parda & 0 & 0 \\
Mulata & $3(13,6)$ & 0 \\
Negra & $6(27,3)$ & $11(50)$ \\
Amarela & $1(4,5)$ & 0 \\
Outros & $10(45,5)$ & $10(45,5)$ \\
Estado civil & & $12(54,5)$ \\
Casado & $14(63,6)$ & $6(27,3)$ \\
Solteiro & $5(22,7)$ & $1(4,5)$ \\
Divorciado & $1(4,5)$ & $2(9,1)$ \\
Viúvo & $2(9,1)$ &
\end{tabular}




\begin{tabular}{|c|c|c|}
\hline \multicolumn{3}{|l|}{ Escolaridade } \\
\hline 10 grau incompleto & $4(18,2)$ & $2(9,1)$ \\
\hline 10 grau completo & 0 & $2(9,1)$ \\
\hline $2^{\circ}$ grau incompleto & $4(18,2)$ & $4(18,2)$ \\
\hline $2^{\circ}$ grau completo & $10(45,4)$ & $11(50)$ \\
\hline Superior incompleto & 0 & $1(4,5)$ \\
\hline Superior completo & $4(18,2)$ & $2(9,1)$ \\
\hline \multicolumn{3}{|l|}{ Situação ocupacional } \\
\hline Dona de casa & $7(31,8)$ & 0 \\
\hline Aposentado & $8(36,4)$ & $8(36,4)$ \\
\hline Autônomo & $3(13,6)$ & $5(22,7)$ \\
\hline Não trabalha, recebe & & \\
\hline benefício & 0 & $3(13,6)$ \\
\hline $\begin{array}{l}\text { Não trabalha, não re- } \\
\text { cebe benefício }\end{array}$ & 0 & $1(4,5)$ \\
\hline Assalariado & $3(13,6)$ & 0 \\
\hline Desempregado & 0 & $2(9,1)$ \\
\hline \multicolumn{3}{|l|}{ Atividade Física } \\
\hline Sim & $10(45 \%)$ & $9(41 \%)$ \\
\hline Não & $12(55 \%)$ & $13(59 \%)$ \\
\hline
\end{tabular}

GE: Grupo experimental; GC: Grupo de controle.

Fonte: Dados da pesquisa

A tabela 3 apresenta as médias, desvios-padrão e valores de $\mathrm{p}$ oriundos do teste $t$ de Student. Após comparar a EVA intra grupos, observou-se melhora da dor no grupo cinesioterapia após o tratamento (Mulligan, $p=0.45$; Exercícios, $p=0.03$ ). Na algometria, não houve diferença (Mulligan $p=0.92$; Exercícios $p=0.38$ ). Também não se verificou melhora da dor após comparação entre os grupos após o tratamento, nem através da EVA nem através da algometria ( $p=0.56, p=0.09$, respectivamente).

Tabela 3 - Média e desvio-padrão da tolerância à dor de acordo com as intervenções e o tempo.

\begin{tabular}{lrrrr}
\hline & \multicolumn{2}{c}{ GE $(\mathbf{n}=\mathbf{2 2})$} & \multicolumn{2}{c}{ GC $(\mathbf{n = 2 2})$} \\
\cline { 2 - 5 } Tolerância à dor & Média $(D P)$ & $(p)$ & Média (DP) & $(p)$ \\
\hline EVN & 5,6 & $(2,7)$ & 5,8 & $(2,7)$ \\
Antes da intervenção & $5,1(3,1)$ & $(0.45)$ & $5,0(3,1)$ & $(0.03)^{*}$ \\
Após intervenção & & & & \\
Algometria & 1,06 & $(0,54)$ & 1,7 & $(0,8)$ \\
Antes da intervenção & $1,06(0,62)$ & $(0.92)$ & $1,5(0,8)$ & $(0.38)$ \\
Após intervenção & &
\end{tabular}

EVN: escala visual numérica da dor, GE: grupo experimental; GC: grupo de controle; DP: desvio padrão; p: valor de p; *valor de $p<0.05$.

Fonte: Dados da pesquisa

\section{DISCUSSÃO}

Nesse estudo pode-se observar que a MWM de Mulligan não foi mais eficaz na redução da dor do ombro se comparada a exercícios terapêuticos, quando reavaliada de forma imediata, tanto medida através da EVA quanto através da algometria. Entretanto, redução da dor foi observada no grupo exercícios após o tratamento $(p=0.03)$ quando mensurada através da EVA.

A EVA permite quantificar a dor através de números. Possui 11 pontos, 0 a 10, onde o ponto 0 (zero) significa nenhuma dor e 10 a pior dor possível. Os demais números representam intensidades moderadas de dor (GALLASCH, 2007). Nesse estudo a média da dor em ambos os grupos foi moderada (Mulligan: 5,6 X Cinesioterapia: 5,8) com redução menor que 1 ponto na escala em ambos os grupos após o tratamento (Mulligan 5,1 X Cinesioterapia: 5,0).

Na mensuração da dor através da algometria, não houve melhora da dor de forma imediata após uma única sessão de tratamento em ambos os grupos. Nossos achados não podem ser comparados com os de Teys, Bisset e Vicenzino (2008) que constataram melhora significativa da dor no ombro através da EVA e pequena redução da dor através da algometria pré e pós-intervenção, ao se comparar a técnica de Mulligan e sham, em pacientes com ombros dolorosos e com restrição de movimentos. Teys et al. (2013), em outro estudo, realizado com 25 indivíduos avaliaram os efeitos da técnica MWM ao longo do tempo, com uma única sessão de tratamento, em indivíduos com dor no ombro, comparando-a ao uso de MWM com tape. Como resultado, observaram que uma única sessão de MWM para dor no ombro reduziu a dor de forma estatisticamente significativa, medida através da EVA.

No estudo de Patrício et al. (2013), os autores também avaliaram os efeitos da MWM na redução da dor, em indivíduos com síndrome do impacto do ombro. 24 indivíduos foram randomizados em dois grupos; um grupo utilizou a técnica MWM, e o placebo realizou os movimentos ativos do ombro, com o fisioterapeuta tocando o ombro, porém não realizando o glide. Os autores concluíram que a técnica de Mulligan reduziu a dor nos indivíduos com síndrome do impacto.

Embora Mulligan advogue a favor da redução imediata da dor após uma única sessão de MWM em ombros dolorosos, nesse estudo não pudemos comprovar essa teoria. A ausência de acompanhamento por tempo mais prolongado pode ser um fator que impediu a observação da eficácia da técnica (MULLIGAN 2003, 2006, 2009). Novos estudos com acompanhamentos a longo prazo são necessários.

Já no estudo de Guimarães, Salvini e Siqueira (2016) não foi encontrada eficácia imediata da MWM em comparação com sham para o aumento da ADM de rotação externa e da abdução, melhora da dor e função do ombro em 27 indivíduos com síndrome do impacto do ombro. Além disso, ambas as técnicas se mostraram ineficazes para ganho de flexão anterior e força muscular.

Os dados obtidos, embora não possam ser considerados representativos da população geral, são relativos à parcela tratada em um serviço de referência de fisioterapia da Universidade Federal da Bahia. As características clínico-demográficas deste estudo apresentam semelhança com estudos anteriores em pacientes com dor no ombro.

Em nosso estudo observamos o predomínio de muIheres. A dor no ombro atinge uma parcela significativa da população, majoritariamente constituída de mulheres, com duração curta ou prolongada. Atinge pessoas em faixas etárias produtivas ou na senescência, por questões 
de envelhecimento biológico (DIAS et al,. 2008; MENDONÇA JÚNIOR; ASSUNÇÃO, 2005) estudaram o perfil da dor no ombro com pacientes portadores da síndrome do manguito rotador e também encontraram predomínio de mulheres. Verificamos, neste estudo, que as mulheres buscam por tratamento mais precocemente que os homens, são mais assíduas e cuidam mais de sua saúde de forma geral, o que pode justificar a maior presença de mulheres em nossa amostra. Além disso, a maioria das mulheres da atualidade assume tripla jornada de trabaIho, uma vez que trabalham fora dois períodos e, quando retornam aos lares à noite, enfrentam as demandas da casa e da família.

A idade média observada neste estudo foi semelhante nos dois grupos: 56 anos no grupo tratado pela técnica de Mulligan e 57 anos no grupo de exercícios terapêuticos. As lesões do manguito rotador constituem causa comum de dor no ombro em pessoas de todas as idades, especialmente naquelas de meia idade, devido ao uso excessivo do ombro (LECLER, 2004). A faixa etária de 40 a 60 anos constitui o período em que as alterações degenerativas tomam proporção maior, com o avançar da idade. Esse fato é associado ao gestual do trabalho, muitas vezes composto por atividades manuais que requerem movimentos repetitivos prolongados, sem pausas e sem ergonomia, e se tornam fatores que predispõem às lesões.

Nossos achados são semelhantes aos da literatura. Dias et al (2008) estudaram a intensidade da dor em pacientes com síndrome do ombro doloroso (SOD) e concluíram que ela foi mais frequente e mais intensa em mulheres, sendo a faixa etária entre 40 e 65 anos a mais acometida. A síndrome do manguito rotador foi também o diagnóstico médico mais frequente em nossa amostra, com maior prevalência entre as mulheres. $O$ excesso de uso do ombro pelas mulheres leva a lesões por repetitividade e impacto. Nosso estudo está de acordo com o que se encontra na prática clínica, onde prevalece o acometimento dos músculos que compõem o manguito rotador. O supraespinhal é o mais acometido, seguido do infraespinhal e subescapular.

Dezessete pacientes do grupo Mulligan se queixaram de dor no ombro direito, $(77,3 \%)$, e 12 (55\%) pacientes do grupo exercícios se queixaram do esquerdo, embora a dominância que prevaleceu foi a da direita, em ambos os grupos. Atividades diárias e ocupacionais podem contribuir para os microtraumas de repetição, o que leva à disfunção do ombro, uma vez que, o ombro dominante é mais requisitado nas atividades.

A maior parte dos pacientes fez algum tipo de exame radiológico, a exemplo de radiografia, ultrassonografia ou ressonância magnética. Por nossa amostra ser composta por pacientes do Sistema Único de Saúde (SUS), os exames de raios- $x$ e ultrassom foram mais frequentes do que a ressonância magnética, que envolve maior custo. Contudo, como muitos pacientes apresentaram envolvimento do manguito ou processo degenerativo articular, tais exames foram suficientes para identificar as estruturas acometidas. Exames como US e a RM são mais específicos no que se refere ao envolvimento das estruturas musculotendíneas do manguito rotador e, atualmente, são mais indicados que a radiografia quando se trata de dor no ombro. Silva (2005), em seu estudo sobre avaliação do ombro por US, identificou a lesão do manguito rotador como a mais frequente, em especial lesão do supraespinhal, em 60 pacientes avaliados. De acordo com Zorzetto et al. 2003 a US é um método acurado de escolha para avaliar a evolução das lesões do ombro, pois observa funcionalmente o manguito rotador com menor custo e maior acessibilidade que outros métodos, sendo método de escolha para avaliação inicial do ombro (ANDRADE et al., 2004; SILVA, 2005; ZORZETTO et al., 2003).

Autoinformada pelos pacientes, a cor da pele predominante foi negra e outras, nos dois grupos. Acreditamos que a influência africana histórica na Bahia, especialmente em Salvador, tenha influenciado esse resultado, porém não se tem conhecimento de relação entre dor no ombro e a cor de pele.

Em relação à escolaridade, pudemos perceber que, como a amostra foi composta por pacientes de baixa renda, menos de $30 \%$ deles tinham o ensino superior completo. $O$ fato de o estudo ter sido realizado em uma clínica-escola de universidade pública favorece um perfil de pacientes de classes sociais mais desfavorecidas, além da localização geográfica de Salvador ser no Nordeste, que, sabidamente, é uma região desfavorecida economicamente.

O tipo de ocupação, muitas vezes, está associado a lesão do ombro e ao afastamento do trabalho. Neste estudo, no grupo do Mulligan, predominaram a ocupação domésticas e a situação de aposentado, enquanto, no grupo de exercícios terapêuticos, predominaram aposentados e autônomos. As atividades domésticas envolvem esforços, como empurrar, carregar peso e alcançar objetos em níveis acima do ombro, ou ainda atividades que requerem a sustentação dos ombros elevados por longos períodos, o que pode levar a lesões nas estruturas musculotendíneas do ombro. No caso de pacientes aposentados, a idade avançada poderia ser um fator que favorece o surgimento de lesões degenerativas musculotendíneas e articulares.

De modo geral, os pacientes deste estudo eram mais sedentários. Observamos que menos da metade realizava algum tipo de atividade física. A maioria praticava caminhada três vezes por semana. Tal atividade não envolve custo e traz benefícios ao complexo do ombro, uma vez que a gravidade traciona o úmero inferiormente, o que reduz o impacto. A decoaptação da articulação glenoumeral promove o aumento da lubrificação articular, além dos movimentos pendulares proporcionados com a dissociação das cinturas escapular e pélvica durante a marcha. Associado a isso, temos também um aumento do aporte sanguíneo para a região do ombro, o que pode melhorar as condições de hipovascularização do músculo supraespinhal, na área de Codman, por meio da oxigena- 
ção proporcionada pelas áreas circunvizinhas (ANDRADE et al., 2004).

\section{CONCLUSÃO}

A MWM não foi mais eficaz que a cinesioterapia na redução da dor do ombro, sendo que a cinesioterapia apresentou mais eficácia quando medida através da EVN e sem melhora significativa ao ser mensurada pela algometria após aplicação de uma única sessão de tratamento.

\section{REFERÊNCIAS}

ANDRADE, R. P. et al. Lesões do manguito Rotador. Rev. Bras. Ortop., São Paulo, v. 39, n. 11/12, 2004. ISSN: 0102-3616.

BRASIL. Ministério da Previdência Social. Coordenação-Geral de Monitoramento Benefício por Incapacidade - CGMBI/DPSSO/SPS/ MPS. 20 Boletim Quadrimestral sobre Benefícios Por Incapacidade. Brasília: DF, 2014.

DIAS et al. Perfil da dor no ombro em pacientes com síndrome do manguito rotador. Rev. Ciênc. Méd. Biol., Salvador, v. 15, n. 3, p. 359362, set./dez. 2016.

DIAS, et al. Intensidade da Dor em pacientes com Síndrome do Ombro Doloroso. Acta Ortop Bras., São Paulo, v. 16, n. 3, p. 165-167, 2008.

GALLASCH CH, Alexandre NMC. The measurement of musculoskeletal pain intensity: a comparison of four methods. Rev Gaúcha Enferm. 2007;28(2):260-5.

GUIMARÃES, J. F.; SALVINI, F.; SIQUEIRA L. Immediate effects of mobilization with movement vs Sham technique on range of motion, strength, and function in patients with shoulder impingement syndrome: randomized clinical trial. J. Manip. Physiol. Ther., Nova York, v.39, n. 9, p. 605-615, 2016.

KISNER, C.; COLBY, L. A. Exercícios Terapêuticos: fundamentos e técnicas. 6 ed. São Paulo: Manole, 2015.

HO C-Y. C.; SOLE, G.; MUNN, J. The effectiveness of manual therapy in the management of musculoskeletal disorders of the shoulder: a systematic review. Man. Ther., Escócia, v. 14, p. 463-474, 2009.

LECLER, A. et al. Incidence of shoulder pain in repetitive work. Occup Environ Med., São Paulo, v. 61, p. 39-44, 2004.

LUIME, J. J. et al. Prevalence and incidence of shoulder pain in the general population; a systematic review. Scand. J Rheumatol., Stockholm , v. 33, n. 2, p. 73-81, 2004.

MENDONÇA JÚNIOR, H. P.; ASSUNÇÃO, A. A. Associação entre distúrbios do ombro e trabalho: breve revisão da literatura. Rev. Bras. Epidemiol., São Paulo, v.8, n.2, p. 167-176, 2005.

MOHER D, Schulz Kf, Altman DG, for the CONSORT Group: The CONSORT statement: revised recommendations for improving the quality of reports of parallel group randomized trials. Lancet, 2001;357(9263):1191-1194

MULLIGAN, B. R. Manual therapy: NAGS, SNAGS, MWMS, etc. 5 ed. Wellington, New Zealand: Plane view Services, 2006. p. 87-108.

MULLIGAN, B. R. The painful dysfunctional shoulder: a new treatment approach using 'mobilization-with-movement'. N. z. j. Physiother., Nova Zelândia, v.31, n.3, p. 140, 2003.

MULLIGAN, B. R. Terapia manual: NAGS, SNAGS, MWM e outras técnicas. $5^{\text {nd }}$ ed. São Paulo: Editorial Premier, 2009. cap. 1. p.9-18/41-50.

PATRÍCIO, R. I. T. Efeitos imediatos da mobilização com movimento na dor, amplitude de movimento e actividade electromiográfica dos músculos da cintura escapular em indivíduos com síndrome do conflito subacromial. 2013. $41 \mathrm{f}$. Dissertação (Mestrado) - Escola Superior de Tecnologia de Saúde do Porto, Porto, 2013.

PAUL, T. M. et al. Central hypersensitivity in patients with subacromialimpingement syndrome. Arch. Phys. Med. Rehabil., Estados Unidos,v. 93, Dec. 2012.

SILVA, M. G. A ultra-sonografia na avaliação da Síndrome do ombro doloroso. 2005. Dissertação (Mestrado em Medicina e Saúde Humana) - Escola Bahiana de Medicina e Saúde Pública (EBMSP), Salvador, 2005.

TEYS, P. et al. One-week time course of the effects of Mulligan's Mobilisation withMovement and taping in painful shoulders Man. Ther., Escócia, v.18, p. 372e377373, 2013.

TEYS, P.; BISSET, L.; VICENZINO B. The initial effects of a Mulligan's mobilization with movement technique on range of movement and pressure pain threshold in pain-limited shoulders. Man. Ther., Escócia, v.13, n.1, 37-42, 2008

VICENZINO, B.; PAUNGMALI, A.; TEYS, P. Mulligan's mobilization-withmovement, positional faults and pain relief: Current concepts from a critical review of literature. Man. Ther., Escócia, v.12, p. 98-108, 2007. DOI:10.1016/j.math.2006.07.012.

WRIGHT, A. Hypoalgesia post-manipulative therapy: A review of a potential neurophysiological mechanism. Man. Ther., Escócia, v.1, p.11-16, 1995.

$\mathrm{XU}, \mathrm{X}$. et al. Chinese cross-cultural adaptation and validation of the Oxford shoulder score. Biomed Central, [s.I.], v. 13, n. 1, 2015.

ZORZETTO, A. A. et al. A Ecografia no diagnóstico das lesões músculotendinosas do ombro. Radiol Bras., v.36, n.4, p.237-242, 2003.
Submetido em: 20/01/2020

Aceito em: 01/09/2020 